\title{
Collective oscillations in a two-dimensional Bose-Einstein condensate with a quantized vortex state
}

\author{
Arup Banerjee \\ Laser Physics Application Section, Centre for Advanced Technology, Indore 452013, India \\ B. Tanatar \\ Department of Physics, Bilkent University, Bilkent 06800 Ankara, Turkey
}

(Received 27 May 2005; published 18 November 2005)

\begin{abstract}
We study the effect of lower dimensional geometry on the frequency splitting of the quadrupole oscillations of a harmonically trapped Bose-Einstein condensate due to the presence of a quantized vortex. To study the effect of two-dimensional geometry we consider a pancake-shaped condensate and employ various models for the coupling parameter depending on the thickness of the condensate relative to the value of the scattering length. Using these models and the sum-rule approach we obtain analytical expressions for the frequency splitting. These expressions are valid for positive scattering length and large $N$. We show that the frequency splitting of the quadrupole oscillations are significantly altered by the reduced dimensionality and also study the evolution of the splitting as the system makes transition from one scattering regime to the other.
\end{abstract}

DOI: $10.1103 /$ PhysRevA.72.053620

\section{INTRODUCTION}

The reduction of dimensionality modifies the physical properties of a Bose-Einstein condensate (BEC) trapped in a harmonic potential significantly in comparison to its threedimensional (3D) counterpart. Many theoretical and experimental studies devoted to the influence of dimensionality on the various properties of BEC have been reported in the literature [1-18]. By making the anisotropy parameter $\lambda$ (defined as the ratio between the frequencies of the harmonic trap in the axial and the transverse directions) much larger than unity $(\lambda \gg 1)$ nearly two-dimensional (2D) flat pancake shaped condensates have been produced in magneto-optical [17] and optical [18] traps. Such pancake-shaped condensates are expected to exhibit the special features of a BEC in reduced dimensions. In the purely $2 \mathrm{D}$ case the scattering properties are quite different as compared to the $3 \mathrm{D}$ case and this in turn leads to significant modification of the bosonboson coupling parameter. For example, the boson-boson coupling parameter in the 2D limit becomes density dependent even at the low density and zero temperature regime. In contrast to this, for the 3D case to the lowest order in density the interactions are described by a constant coupling strength and the deviations occur only when the quantum depletion and the finite temperature effects are taken into consideration [19].

It is well known that the phenomenon of superfluidity is strongly related to the phenomenon of Bose-Einstein condensation. The quantized vortex states play an important role in establishing the superfluid nature of the condensates. Therefore, it is natural to expect that the vortex states will also play a crucial role in understanding and characterizing the superfluid nature of the condensates in lower dimensions also. However, in an experiment direct observation of vortex in a trapped gas is difficult due to the small size of the vortex core in comparison to the size of the condensate. This problem has been circumvented by observing the free expansion of the cloud. When the cloud is allowed to expand freely
PACS number(s): 03.75.Kk, 05.30.Jp, 34.50.-s, 67.40.Db

after the trap has been switched off, the size of the vortex core increases faster than that of the condensate cloud which then allows one to distinguish the free expansion of a cloud with a quantized vortex from that of without any vortex state. Realizing the importance of vortex states in lower dimensional condensates studies on the effects of the boson-boson coupling parameter on the ground-state density of the condensate with a vortex state $[9,11]$, free expansion of a cloud with a vortex [14] and the critical angular frequency needed for the creation a low angular momentum vortex state [15] have already been carried out.

The measurements of frequencies of the collective oscillations also serve as a way to detect the vortex states in the BEC. For example, the presence of a quantized vortex state leads to the splitting of the frequencies of the two modes of quadrupole oscillations with opposite values of the third component of the angular momentum, which are degenerate in the absence of a quantized vortex state [20-23]. The vortex state in a condensate breaks the time reversal symmetry which in turn results in the removal of the degeneracy of the two modes of oscillations carrying opposite values of angular momentum. It is well known that the measurement of the frequencies of the collective oscillations can be carried out with very high precision. Therefore, the splitting of the two quadrupole modes of collective oscillations can be employed to detect the presence of a quantized vortex state in a BEC with quite high accuracy [23]. This has motivated us to study the effect of the lower dimensionality on the splitting of two degenerate quadrupole modes due to the presence of a vortex state. To this end we investigate the effect of different models for the boson-boson coupling parameter, simulating the inter-boson interaction in reduced dimensions, on the splitting of the degenerate quadrupole modes of collective oscillations in the BEC with a quantized vortex state.

The paper is organized as follows. We briefly describe the models of boson-boson coupling parameters corresponding to different scattering regimes and derive the expressions for the splitting in the frequencies of quadrupole modes caused 
by a quantized vortex by sum-rule approach of many-body response theory in Sec. II. In Sec. III we present and discuss our results. The paper is concluded in Sec. IV.

\section{FORMULATION}

\section{A. Models}

We consider a dilute condensate with $N$ bosons confined in an anisotropic (pancake shaped) harmonic trap characterized by the frequencies $\omega_{\perp}$ and $\omega_{z}=\lambda \omega_{\perp}$ with the anisotropy parameter $\lambda$ being much larger than unity. For such a highly anisotropic trap the motion in the $z$-direction is frozen and the state of the condensate can be described by wave function $\Psi(\mathbf{r})$ in $\{x, y\}$ plane. The wave function $\Psi(\mathbf{r})$ can be determined by minimizing the local density energy functional

$$
E[\Psi]=\int d^{2} \mathbf{r}\left(\frac{\hbar^{2}}{2 m}|\nabla \Psi|^{2}+v_{\mathrm{ext}}(\mathbf{r})|\Psi|^{2}+\epsilon(\rho)|\Psi|^{2}\right),
$$

where the density $\rho=|\Psi|^{2}$ and $v_{\text {ext }}(\mathbf{r})$ is the external harmonic potential in the transverse direction given by

$$
v_{\text {ext }}(\mathbf{r})=\frac{1}{2} m \omega_{\perp}^{2}\left(x^{2}+y^{2}\right) .
$$

In the above equation [Eq. (1)] the first and the third terms represent the kinetic energy and the energy due to the interatomic interaction within local density approximation (LDA), respectively. The above energy functional can be generalized to describe the condensate with a quantized vortex state $[9,24]$. For this purpose we consider a condensate with a vortex at the center of the trap. The wave function of such a state can be written as

$$
\Psi(\mathbf{r})=\psi(r) e^{i \kappa \phi},
$$

where $r=\sqrt{x^{2}+y^{2}}, \phi$ is the angle around the vortex core and $\kappa$ is an integer denoting the quantum circulation. Substituting the above complex wave function in Eq. (1) we obtain the generalized energy functional for the condensate with a vortex state as $[9,24]$

$$
E[\psi]=\int d^{2} \mathbf{r}\left(\frac{\hbar^{2}}{2 m}|\nabla \psi|^{2}+\frac{\hbar^{2} \kappa^{2}}{2 m r^{2}}|\psi|^{2}+v_{\mathrm{ext}}(\mathbf{r})|\psi|^{2}+\epsilon(\rho)|\psi|^{2}\right) .
$$

In the above equation $\rho(r)=|\psi(r)|^{2}$ and the presence of centrifugal term due to the vortex state makes the above functional different from Eq. (1). The minimization of the above functional with respect to $\psi(r)$ with the constraint condition

$$
\int \mid \psi(r) d^{2} \mathbf{r}=N
$$

leads to the Gross-Pitaevskii equation for the condensate with vortex state $[14,24]$

$$
\left(-\frac{\hbar^{2}}{2 m} \nabla^{2}+\frac{\hbar^{2} \kappa^{2}}{2 m r^{2}}+\frac{1}{2} m \omega_{\perp}^{2} r^{2}+\frac{\partial[\epsilon(\rho) \rho]}{\partial \rho}\right) \psi(r)=\mu \psi(r),
$$

where $\mu$ is the chemical potential. The wave function $\psi(r)$ for the condensate with a single vortex is determined by solving the above nonlinear Schrödinger equation.

In order to determine the wave function of the condensate we need to know the form of interatomic interaction energy $\epsilon(\rho)$. In the following we discuss the forms of $\epsilon(\rho)$ employed in this paper to study the effect of lower dimensionality on the splitting of the two quadrupole modes of collective oscillations. Within the local density approximation the interaction energy per particle $\epsilon(\rho)$ is given by

$$
\epsilon(\rho)=\frac{g}{2} \rho(\mathbf{r}),
$$

where $g$ is the coupling constant whose form depends on the collisional properties of the condensate. For example, $g$ is independent of the density for the 3D case, on the other hand, in the purely $2 \mathrm{D}$ regime it depends logarithmically on the density. In the following we briefly describe the models for the coupling constant $g$ valid in different collisional regimes.

For the 3D system the coupling parameter $g$ which is a constant and completely determined by the $s$-wave scattering length $a$ given by

$$
g=\frac{4 \pi \hbar^{2}}{m} a .
$$

When the linear dimension $a_{z}$ of the condensate along the $z$-direction is much larger than the 3D scattering length $a\left(a_{z} \gg a\right)$, the collisions still take place in three dimensions. Under this condition the effective coupling constant which includes the effects of reduced dimensionality is expressed as $g_{\mathrm{Q} 3 \mathrm{D}}=g\left|\phi_{0}(0)\right|^{2}$ where $g$ is the 3D coupling constant given above and $\phi_{0}(0)=\left(2 \pi a_{z}^{2}\right)^{-1 / 4}$ is the axial ground state wave function at $z=0$. More explicitly $[4,10,12,25]$,

$$
g_{\mathrm{Q} 3 \mathrm{D}}=2 \sqrt{2 \pi} \frac{\hbar^{2} a}{m a_{z}} .
$$

On further increasing the anisotropy and simultaneously $a_{z}$ becoming comparable to $a\left(a_{z} \approx a\right)$, the collisions between the bosons start getting affected by the tight confinement along the $z$-direction. Under such a condition a condensate is said to be in the quasi-two-dimensional (Q2D) regime. The coupling constant in this regime is given by

$$
g_{\mathrm{Q} 2 \mathrm{D}}=\frac{2 \sqrt{2 \pi} \frac{\hbar^{2} a}{m a_{z}}}{1+\frac{a}{\sqrt{2 \pi} a_{z}}\left|\ln \left[2(2 \pi)^{3 / 2} \rho(\mathbf{r}) a a_{z}\right]\right|} .
$$

Here $\rho(\mathbf{r})$ is the ground state density of the condensate. The above expression was originally derived by Petrov et al. $[5,6]$ by studying the scattering properties of a bosonic system which is trapped harmonically in the $z$-direction and uniform in the $\{x, y\}$ plane. A similar expression was later derived by Lee et al. [9] employing the many-body $T$-matrix approach. It is important to note here that in the Q2D regime the coupling constant becomes dependent on the density in accordance with the behavior of collisions in two dimensions. Finally, when $a_{z}$ becomes much smaller than $a\left(a_{z}\right.$ $\ll a$ ), the collisions can be safely assumed to be taking place 
in two dimensions resulting in a $2 \mathrm{D}$ condensate. The coupling constant in the $2 \mathrm{D}$ regime is given by

$$
g_{2 \mathrm{D}}=\frac{4 \pi \hbar^{2}}{m} \frac{1}{\left|\ln \rho(\mathbf{r}) a^{2}\right|} .
$$

Notice that the expression for $g_{2 \mathrm{D}}$ also depends on the density $\rho(\mathbf{r})$ but the information about the confinement direction is absent as it corresponds to the purely $2 \mathrm{D}$ case. The expression for $g_{2 \mathrm{D}}$ was derived in Ref. [26] for a homogenous Bose gas of hard disks. This form of $g_{2 \mathrm{D}}$ has been employed to study the properties confined bosons in two-dimensions $[3,7]$ and the rigorous justification for this use was provided by Lieb et al. [8]. We will employ these expressions for the boson-boson coupling parameter to study the effect of reduced dimensionality on the splitting of frequencies of the degenerate quadrupole modes of collective oscillations of harmonically trapped Bose gas with a quantized vortex state.

\section{B. Sum-rule approach for collective oscillations}

Having described the models for the coupling constant in different scattering regimes, we now describe the sum-rule approach of many-body response theory for calculation of frequencies of collective oscillations. In the collisionless regime the collective excitation frequencies of a confined bosonic gas is well described by the sum rule approach. The most important advantage of this approach is that the calculation of frequencies requires the knowledge of the groundstate wave function (or the ground-state density) of the many-body system only. This method has been extensively applied to calculate the frequencies of the collective oscillations of trapped atomic gases [27]. In this paper we follow the sum-rule approach developed in Ref. [20] for the calculation of the splitting of the quadrupole modes of collective oscillations. The collective excitations of any many-body system are generally probed by applying an external excitation field. For excitation operators $F_{+}$and $F_{-}$exciting two quadrupole modes with opposite angular momentum, respectively, the corresponding strength functions are given by $[28,29]$

$$
S_{ \pm}(E)=\sum_{n}\left|\left\langle 0\left|F_{ \pm}\right| n\right\rangle\right|^{2} \delta\left(E-E_{n}\right),
$$

where $|0\rangle$ denotes the ground state of the system and $E_{n}$ $-E_{0}$ is the excitation energy of the eigenstate $|n\rangle$ of the Hamiltonian $H$ relative to the ground state energy $E_{0}$. The $p$ th order moments of the strength function can be defined as

$$
m_{p}^{ \pm}=\int E^{p}\left[S_{+}(E) \pm S_{-}(E)\right] d E .
$$

These moments provide various energy weighted sum rules which are employed to obtain the frequencies of collective oscillations. One of the important properties of these moments is that, for a given $p$, some of the moments can be expressed in terms of the commutators of the excitation operators with the many-body Hamiltonian $H$. Some of the en- ergy weighted sum rules which are relevant for this paper are

$$
\begin{gathered}
m_{0}^{-}=\left\langle 0\left|\left[F^{\dagger}, F\right]\right| 0\right\rangle, \\
m_{1}^{+}=\left\langle 0\left|\left[F^{\dagger},[H, F]\right]\right| 0\right\rangle, \\
m_{2}^{-}=\left\langle 0\left|\left[\left[F^{\dagger}, H\right],[H, F]\right]\right| 0\right\rangle, \\
m_{3}^{+}=\left\langle 0\left|\left[\left[F^{\dagger}, H\right],[H,[H, F]]\right]\right| 0\right\rangle,
\end{gathered}
$$

where [,] represents the commutator between the corresponding operators.

These expressions can also be used to calculate the frequency shifts of the quadrupole oscillations of a trapped condensate in reduced dimensions. For the purpose of computation we first need to choose the appropriate excitation operators. In two dimensions the quadrupole excitation operators can be written as

$$
F_{ \pm}=(x \pm i y)^{2}=r^{2} e^{ \pm 2 i \theta}
$$

carrying angular momentum \pm 2 . Using these excitation operators, the moments of Eq. (10) can be evaluated and they are given by $[20,30]$

$$
\begin{gathered}
m_{0}^{-}=0, \\
m_{1}^{+}=\frac{8 \hbar^{2}}{m}\left\langle 0\left|x^{2}+y^{2}\right| 0\right\rangle, \\
m_{2}^{-}=\frac{16 \hbar^{3}}{m^{2}}\left\langle 0\left|x p_{y}-y p_{x}\right| 0\right\rangle, \\
m_{3}^{+}=\frac{16 \hbar^{4}}{m}\left(\left\langle 0\left|x^{2}+y^{2}\right| 0\right\rangle+\frac{\left\langle 0\left|p_{x}^{2}+p_{y}^{2}\right| 0\right\rangle}{m^{2} \omega_{\perp}^{2}}\right),
\end{gathered}
$$

where $p_{i}$ is the $i$ th component of the linear momentum vector p. In deriving the above equations the relation $F_{+}^{\dagger}=F_{-}$has been used. It is worth noticing that the moments up to thirdorder do not depend on the boson-boson coupling parameter (two-body interaction) explicitly. This is because the energies within the LDA do not contribute to moments up to third order for the excitation operators satisfying $\nabla^{2} F_{ \pm}=0$ [31].

In order to use the above expressions for the calculation of the shift in frequencies of quadrupole modes we now assume that the moments $m_{p}^{ \pm}$are exhausted by a single excitation with frequency $\omega_{ \pm}$and strength $\sigma_{ \pm}$. Under such singlemode approximation the strength distribution can be written as

$$
S_{ \pm}(E)=\sigma_{ \pm} \delta\left(E-\hbar \omega_{ \pm}\right) .
$$

The vanishing of $m_{0}^{-}$moment leads to the result $\sigma_{+}=\sigma_{-}=\sigma$. We note here that the single mode approximation is well suited for the condensates with large number of atoms $N$ and positive scattering length $a$ [20]. Using Eqs. (17) and (13) we obtain the following expression for the difference between the two frequencies: 


$$
\hbar \delta=\hbar\left(\omega_{+}-\omega_{-}\right)=\frac{m_{2}^{-}}{m_{1}^{+}} .
$$

Furthermore, the individual frequencies of the quadrupole oscillations can be determined by

$$
\hbar^{2}\left(\frac{\omega_{+}+\omega_{-}}{2}\right)^{2}=\frac{m_{3}^{+}}{m_{1}^{+}}-\frac{3}{4}(\hbar \delta)^{2} .
$$

In this paper we use Eqs. (16), (18), and (19) to determine the frequency for the splitting of the two quadrupole modes and also the frequencies of each mode separately. As mentioned above albeit the frequencies do not depend on the boson-boson coupling parameter explicitly but implicit dependence on the two-body interaction enter through the ground-state wave function or the density of the condensate which crucially depend on the nature of the boson-boson interaction. In the next section we present the results of our calculation showing the effect of reduced dimensionality on the splitting of the two quadrupole modes of collective oscillations.

\section{RESULTS AND DISCUSSION}

To study the effect of the reduced dimensionality on the splitting of the two quadrupole modes first we need to calculate the three moments $m_{1}^{+}, m_{2}^{-}$, and $m_{3}^{+}$. To this end we calculate the wave functions (or densities) of the Q3D, Q2D and the $2 \mathrm{D}$ condensates by solving Eq. (6) within the Thomas-Fermi (TF) approximation. Furthermore, for Q2D and $2 \mathrm{D}$ cases the TF ground-state densities are obtained by neglecting the spatial dependence of the coupling constant. This is achieved by using the results of the homogenous bosonic system to relate the density to the chemical potential. It has been shown in Refs. $[9,10]$ that for large $N$ the TF approximation and the spatially independent form of the coupling constant yield sufficiently accurate results. We note here that in this paper the number of bosons $N$ in the condensate is chosen such that both the single-mode and the TF approximations are well satisfied by the condensate. Within the TF approximation we obtain the following expressions for the splitting of frequencies (in units of $\omega_{\perp}$ ) of the quadrupole modes excited by the operator $F_{+}$and $F_{-}$in different scattering regimes:

$$
\begin{gathered}
\delta_{2 \mathrm{D}}=\frac{12 \kappa N}{\ln \left(\frac{\tilde{\mu}_{2 D} \tilde{a}^{2}}{4 \pi \lambda}\right) \tilde{\mu}_{2 \mathrm{D}}^{3},} \\
\delta_{\mathrm{Q} 2 \mathrm{D}}=\frac{12 \kappa N}{\left[\sqrt{2 \pi} \tilde{a}^{-1}+\ln \left(\frac{\lambda}{2 \pi \tilde{\mu}_{Q 2 \mathrm{D}}}\right)\right] \tilde{\mu}_{\mathrm{Q} 2 \mathrm{D}}^{3}}, \\
\delta_{\mathrm{Q} 3 \mathrm{D}}=\frac{12 \kappa N \tilde{a}}{\sqrt{2 \pi} \tilde{\mu}_{\mathrm{Q} 3 \mathrm{D}}^{3}},
\end{gathered}
$$

where $N$ is the total number of particles in the condensate, $\kappa$ represents the quantized angular momentum per particle which can assume values $\kappa=1,2,3 \ldots, \tilde{\mu}_{i}(i=2 \mathrm{D}, \mathrm{Q} 2 \mathrm{D}$, and $\mathrm{Q} 3 \mathrm{D})$ denotes the chemical potential of the respective condensate in units of $\hbar \omega_{\perp}$ and the dimensionless parameter $\tilde{a}$ $=a / a_{z}$. The chemical potential $\tilde{\mu}$ is determined by normalizing the corresponding TF density to the total number of particles $N$. In obtaining Eq. (20) we have neglected the terms of the order $O\left[1 /(2 \tilde{\mu})^{2}\right]$ which arise due to the centrifugal term in Eq. (6). For large $N$ corrections introduced by these terms are negligible. Next, using Eqs. (19) and (16) the expression for the individual frequencies within the TF approximation can be written as

$$
\omega_{ \pm}=\sqrt{2} \omega_{\perp} \pm \delta
$$

The above two equations [Eqs. (20) and (21)] constitute the main results of this paper and in the following we discuss these results. Before proceeding with the discussion of our results we compare the result of the frequency shift for the Q3D model with the corresponding result for the 3D case. The 3D expression for the frequency shift within the TF approximation is given by [20]

$$
\delta_{3 \mathrm{D}}=\frac{7 \kappa}{\lambda^{1 / 5}}(15 N \widetilde{a})^{-2 / 5}
$$

By substituting the TF result for the $\tilde{\mu}_{\mathrm{Q} 3 \mathrm{D}}$ given by

$$
\tilde{\mu}_{\mathrm{Q} 3 \mathrm{D}}=(\sqrt{8 / \pi} N \widetilde{a})^{1 / 2}
$$

in the expression for $\delta_{\mathrm{Q} 3 \mathrm{D}}$ we get

$$
\delta_{\mathrm{Q} 3 \mathrm{D}}=\frac{12 \kappa}{\sqrt{2 \pi}(8 / \pi)^{3 / 4}}(N \widetilde{a})^{-1 / 2} .
$$

The above two equations [Eqs. (22) and (24)] show that $\delta_{3 \mathrm{D}}$ exhibits different scaling with respect to $N \tilde{a}$ as compared to $\delta_{\mathrm{Q} 3 \mathrm{D}}$ and the multiplicative factors are also different. The differences between $\delta_{3 \mathrm{D}}$ and $\delta_{\mathrm{Q} 3 \mathrm{D}}$ arise due to reduced dimensionality in the Q3D case. We now discuss the results for a pancake shaped condensate similar to the one achieved in the experiment of Görlitz et al. [17] with ${ }^{23} \mathrm{Na}$ atoms. In accordance with the experiment these parameters are $N$ $=10^{5}, \lambda=26.33$, and $\tilde{a}=3.8 \times 10^{-3}$. We note here that this value of $N$ is in conformity with the TF approximation. These parameters indicate that the condensate produced in the above experiment falls within the Q3D regime. From Eq. (20) with above parameters and for one quantum circulation $(\kappa=1)$ we obtain $\delta_{2 \mathrm{D}}=1.625 \times 10^{-2}, \delta_{\mathrm{Q} 2 \mathrm{D}}=0.1217$ and $\delta_{\mathrm{Q} 3 \mathrm{D}}$ $=0.1218$ in units of $\omega_{\perp}$. These results clearly show that for the condensates achieved in the experiment of Görlitz et al., the $2 \mathrm{D}$ model underestimates the frequency of splitting between the two quadrupole modes quite significantly in comparison to the corresponding values for Q3D and Q2D models. On the other hand, both Q3D and Q2D models give the same numbers for the splitting. This is consistent with the fact that for the above-mentioned parameters, the system undergoes collisions in 3D but has two-dimensional characteristic with respect to the confinement effects. Now to study 
TABLE I. Frequency splitting of the two quadrupole modes carrying opposite angular momentum due to the presence of a single quantized vortex with $\kappa=1$ in units of $\omega_{\perp}$ for three different values of the dimensionless interaction parameter $\tilde{a}=a / a_{z}$ calculated using Eq. (20) for $N=10^{5}$ and $\lambda=2 \times 10^{5}$.

\begin{tabular}{cccc}
\hline \hline$\tilde{a}$ & $\delta_{Q 3 \mathrm{D}}$ & $\delta_{\mathrm{Q} 2 \mathrm{D}}$ & $\delta_{2 \mathrm{D}}$ \\
\hline $3.8 \times 10^{-3}$ & 0.122 & 0.122 & $2.17 \times 10^{-2}$ \\
0.33 & $1.31 \times 10^{-2}$ & $1.70 \times 10^{-2}$ & $1.63 \times 10^{-2}$ \\
2.68 & $4.59 \times 10^{-2}$ & $1.14 \times 10^{-2}$ & $1.28 \times 10^{-2}$ \\
\hline \hline
\end{tabular}

the effect of different models of the coupling constant and their applicability we choose three different values of the parameter $\widetilde{a}, \widetilde{a}=3.8 \times 10^{-3}, 0.33$, and 2.68 . These values are chosen such that the first, second, and third values of $\widetilde{a}$ fall in the Q3D, Q2D, and the 2D regimes, respectively. In addition to this we also choose $\lambda=2 \times 10^{5}$ so that the condensate has negligible extension in the $z$-direction and the motion along this axis is completely frozen. The results with these parameters are presented in Table I. Again, we can observe from Table I that for $\widetilde{a}=3.8 \times 10^{-3}$ the values predicted by the Q3D and the Q2D models are the same and the corresponding result from the 2D model is quite lower than these two values. For $\tilde{a}=0.33$ the numbers obtained by both $\mathrm{Q} 2 \mathrm{D}$ and 2D models are very close to each other but markedly different from the result of the Q3D model. As discussed above, for $\tilde{a}=0.33$ along with the large value of $\lambda$ the scattering properties start to get influenced by the confinement. In this situation it is anticipated that both Q2D and 2D models will give significantly different values in comparison to the corresponding Q3D results. In the light of our earlier discussion we expect that for $\widetilde{a}=0.33$ the coupling constant is better described by the Q2D model. As the interaction parameter $\widetilde{a}$ is further increased to a value $\widetilde{a}=2.68$ the scattering properties become truly two dimensional, consequently for this value of $\widetilde{a}$ the $2 \mathrm{D}$ model should be more appropriate for the description of the condensate. In contrast to the case of $\tilde{a}$ $=0.33$, for $\widetilde{a}=2.68$ the value of the splitting obtained by the 2D model is higher than the corresponding number from the Q2D model.

Finally, to illustrate the importance of scattering mechanisms in various regimes, we plot in Fig. 1 the splitting of the quadrupole modes obtained with three different models as a function of the interaction parameter $\widetilde{a}$. The curves are drawn with the anisotropy parameter $\lambda=2 \times 10^{5}$ and the number of atoms $N=10^{5}$. It can be clearly seen from Fig. 1 that the amount of splitting of the quadrupole modes obtained with the three models of the coupling constant exhibit distinctly different trends with increasing interaction parameter $\widetilde{a}$. As mentioned before it is only for $\widetilde{a} \ll 1$ the values of splitting obtained by the Q3D and the Q2D models are nearly the same but in the same regime $2 \mathrm{D}$ model gives quite different values. On the other hand, when $\widetilde{a}$ exceeds the value 0.1 , the effects of reduced dimensionality start influencing the scattering properties and both Q2D and 2D models give different results as compared to the Q3D numbers. Therefore, we conclude from our results that the splitting of the frequencies of two quadrupole modes carrying opposite

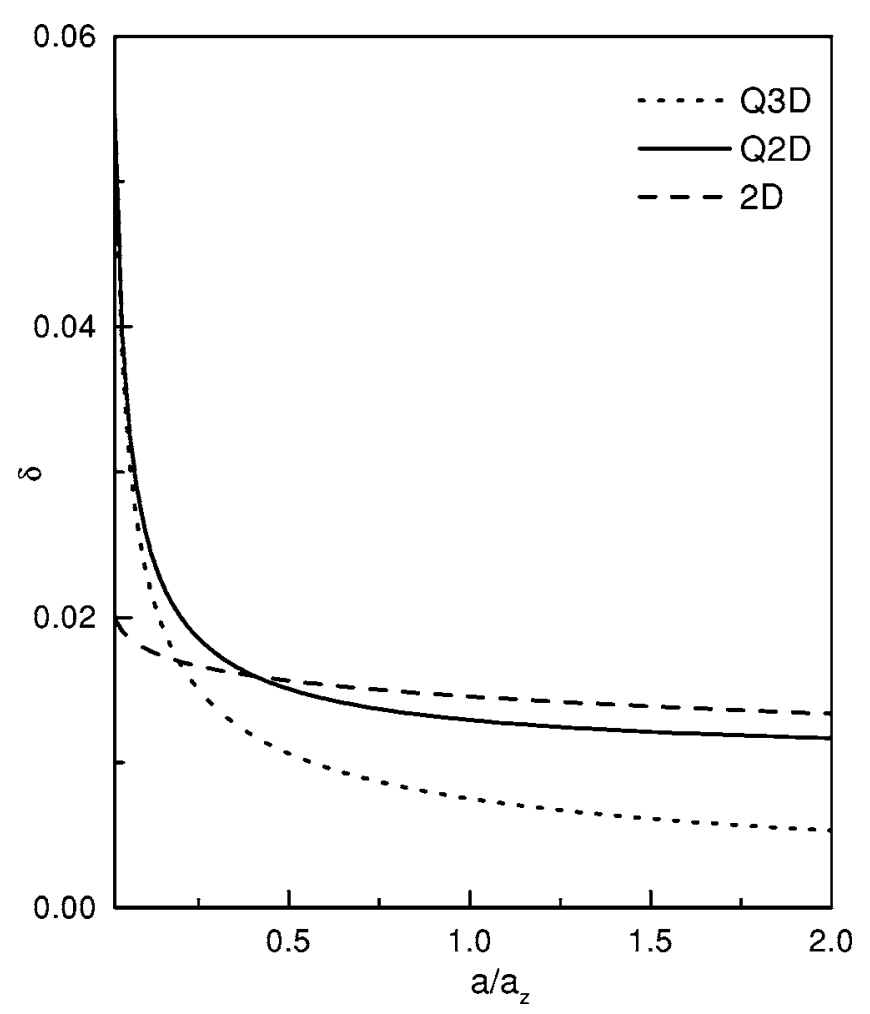

FIG. 1. Frequency splitting $\delta$ (in units of $\omega_{\perp}$ ) of the quadrupole modes of $10^{5}{ }^{23} \mathrm{Na}$ atoms confined in a highly deformed trap with $\lambda=2 \times 10^{5}$ with a single quantized vortex for $\kappa=1$ as a function of the interaction parameter $\tilde{a}=a / a_{z}$. The solid line represents results for the Q2D (geometric confinement, 2D scattering) case while the corresponding $2 \mathrm{D}$ results are shown by the dashed line and the $\mathrm{Q} 3 \mathrm{D}$ (geometric confinement, 3D scattering) results are displayed by the dotted line.

angular momentum can be used to identify the dimensionality of the systems as the system evolves from Q3D to a strictly $2 \mathrm{D}$ regime. These results can also be used to test the validity of different models of the coupling constant by comparing them with the experimental values.

\section{CONCLUSION}

We have calculated the frequency splitting the quadrupole modes of collective oscillations carrying opposite angular momentum of a condensate confined in a highly anisotropic trap due to the presence of a quantized vortex. The condensate is tightly trapped along the $z$-axis such that the motion along this axis is frozen. For such a condensate depending on the value of the ratio between the size of the condensate along the $z$-direction and the s-wave scattering length, three different regimes can be identified. These three different regimes are described by three different models for the bosonboson interactions. We have calculated the frequency splitting of the quadrupole oscillations and studied its dependence on the boson-boson coupling model. For this purpose we have used the sum-rule approach of many-body response theory along with the ground-state density obtained within the TF approximation. The main result of this paper is that the different models for the coupling constant affect 
splitting frequency in a significant way. The effect of modification of the collisional properties in two dimensions start altering the value of frequency splitting when the parameter $\tilde{a}$ becomes more than 0.1 . It would be interesting to have systematic experiments in the parameter regime considered in this work.

\section{ACKNOWLEDGMENTS}

B.T. thanks the Turkish Academy of Sciences (TUBA) and the Scientific and Technical Research Council of Turkey (TUBITAK) for partial support.
[1] V. Bagnato and D. Kleppner, Phys. Rev. A 44, 7439 (1991).

[2] W. J. Mullin, J. Low Temp. Phys. 106, 615 (1997).

[3] S. I. Shevchenko, Sov. Phys. JETP 73, 1009 (1991).

[4] T.-L. Ho and M. Ma, J. Low Temp. Phys. 115, 61 (1999).

[5] D. S. Petrov, M. Holzmann, and G. V. Shlyapnikov, Phys. Rev. Lett. 84, 2551 (2000).

[6] D. S. Petrov and G. V. Shlyapnikov, Phys. Rev. A 64, 012706 (2001).

[7] E. B. Kolomeisky, T. J. Newman, J. P. Straley, and X. Qi, Phys. Rev. Lett. 85, 1146 (2000).

[8] E. H. Lieb, R. Seiringer, and J. Yngvason, Commun. Math. Phys. 224, 17 (2001).

[9] M. D. Lee, S. A. Morgan, M. J. Davis, and K. Burnett, Phys. Rev. A 65, 043617 (2002).

[10] B. Tanatar, A. Minguzzi, P. Vignolo, and M. P. Tosi, Phys. Lett. A 302, 131 (2002).

[11] B. Tanatar, J. Phys. B 35, 2719 (2002).

[12] L. Salasnich, A. Parola, and L. Reatto, Phys. Rev. A 65, 043614 (2002).

[13] T. K. Ghosh and S. Sinha, Eur. Phys. J. D 19, 371 (2002).

[14] O. Hosten, P. Vignolo, A. Minguzzi, B. Tanatar, and M. P. Tosi, J. Phys. B 36, 2455 (2003).

[15] K. K. Rajagopal, B. Tanatar, P. Vignolo, and M. P. Tosi, Phys. Lett. A 328, 500 (2004).

[16] A. Banerjee, Phys. Lett. A 332, 291 (2004).

[17] A. Görlitz, J. M. Vogels, A. E. Leanhardt, C. Raman, T. L. Gustavson, J. R. Abo-Shaeer, A. P. Chikkatur, S. Gupta, S.
Inouye, T. Rosenband, and W. Ketterle, Phys. Rev. Lett. 87, 130402 (2001).

[18] N. L. Smith, W. H. Heathcote, G. Hechenblaikner, E. Nugen, and C. J. Foot, J. Phys. B 38, 223 (2005).

[19] D. A. W. Hutchinson, R. J. Dodd, and K. Burnett, Phys. Rev. Lett. 81, 2198 (1998).

[20] F. Zambelli and S. Stringari, Phys. Rev. Lett. 81, 1754 (1998).

[21] A. A. Svidzinsky and A. L. Fetter, Phys. Rev. A 58, 3168 (1998).

[22] M. Guilleumas and R. Graham, Phys. Rev. A 64, 033607 (2001).

[23] P. C. Haljan, I. Coddington, P. Engels, and E. A. Cornell, Phys. Rev. Lett. 87, 210403 (2001).

[24] F. Dalfovo and S. Stringari, Phys. Rev. A 53, 2477 (1996).

[25] R. K. Bhaduri, S. M. Reimann, S. Viefers, A. G. Choudhury, and M. K. Srivastava, J. Phys. B 33, 3895 (2000).

[26] M. Schick, Phys. Rev. A 3, 1067 (1971).

[27] L. Pitaevskii and S. Stringari, Bose-Einstein Condensation (Clarendon, Oxford, 2003).

[28] O. Bohigas, A. M. Lane, and J. Martorell, Phys. Rep. 51, 267 (1979).

[29] E. Lipparini and S. Stringari, Phys. Rep. 175, 103 (1989).

[30] M. Modugno, L. Pricoupenko, and Y. Castin, Eur. Phys. J. D 22, 235 (2003).

[31] L. Serra, F. Garcias, M. Barranco, N. Barberan, and J. Navarro, Phys. Rev. B 41, 3434 (1990). 